\title{
Rarefied Background Flow in a Vacuum Chamber
}

\author{
Chunpei Cai ${ }^{*}$ Iain D. Boyd ${ }^{\dagger}$ and Quanhua Sun ${ }^{\ddagger}$ \\ Department of Aerospace Engineering \\ University of Michigan, Ann Arbor, MI 48109
}

\begin{abstract}
Spacecraft propulsion systems, such as Hall thrusters, are designed and tested in large vacuum chambers. The pumping capacity of modern facilities makes it possible to maintain pressures as low as $10^{-3}-10^{-4} \mathbf{P a}$. In this study, several free molecular models are developed to analyze the background flow inside a vacuum chamber. These models lead to various sets of analytical results including velocity distribution functions for the background flow and formulas to compute the vacuum pump absorption coefficient. In the present study, the models are applied to analyze the rarefied background flow in a specific vacuum chamber. The results indicate that, with specific parameters, the background flow can have a significant nonzero mean velocity and cannot be considered to have a Maxwellian velocity distribution. Several modifications of the background flow treatment for numerical simulations are proposed with the aid of these analytical results.
\end{abstract}

\section{Nomenclature}

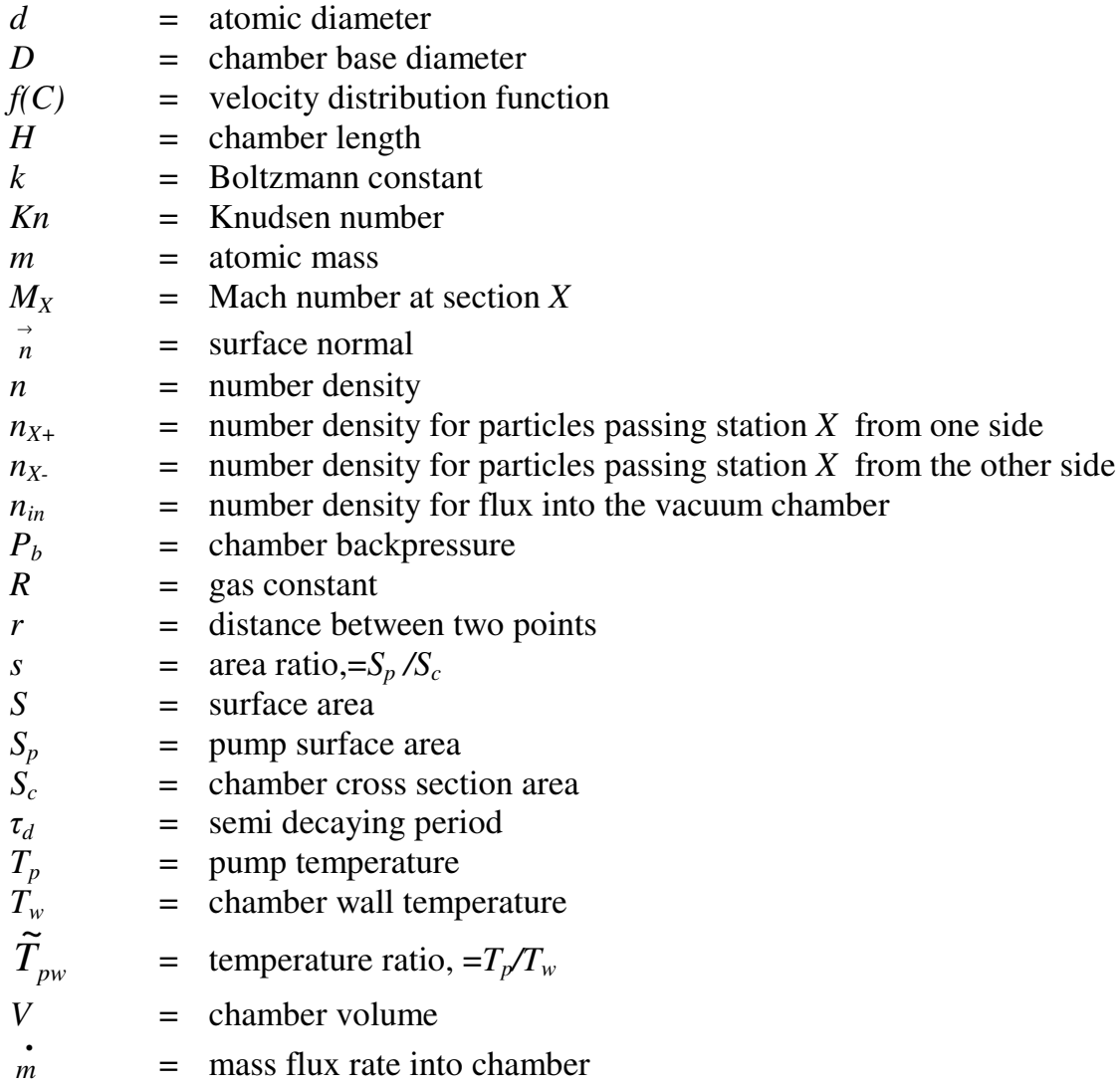

\footnotetext{
* Graduate student, AIAA student member (ccai@engin.umich.edu).

${ }^{\dagger}$ Professor, AIAA associate fellow(iainboyd@umich.edu).

* Research associate, AIAA senior member(qsun@engin.umich.edu).

Copyright $\odot 2005$ by the American Institute of Aeronautics and Astronautics, Inc., All Rights Reserved.
} 


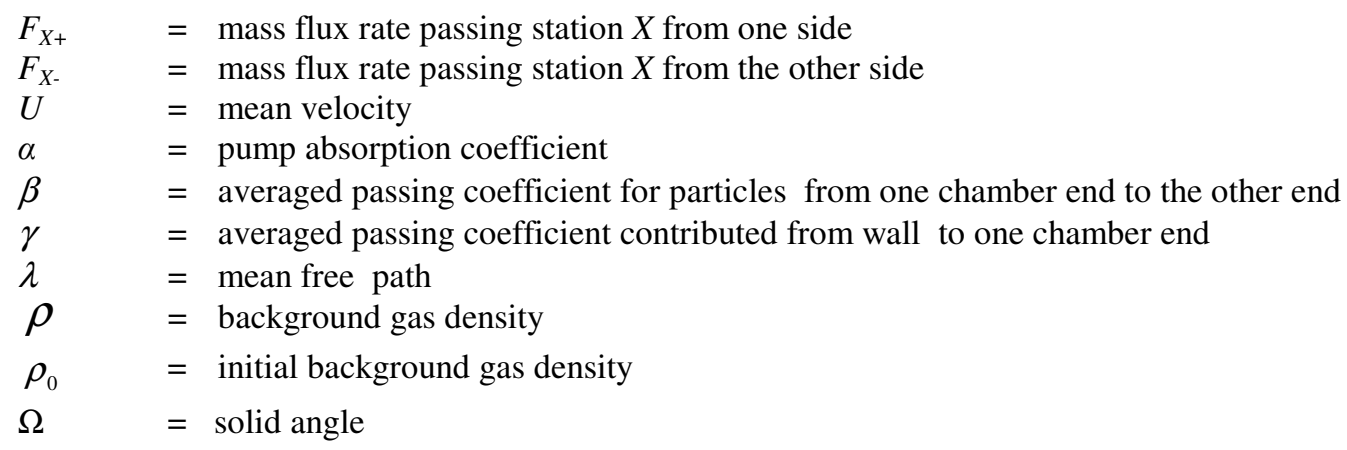

\section{Introduction}

$\mathrm{V}$

ACUUM chambers have wide application for a variety of purposes such as materials processing and electric propulsion experiments. The goal of vacuum chambers is to maintain a low pressure. For example, in the experiments of testing a cluster of high power electric plasma thrusters inside vacuum chambers, ${ }^{1-3}$ the backpressure was maintained at about $10^{-3}-10^{-4} \mathrm{~Pa}$. In such experiments, a high backpressure will distort the exhaust plume flow and affect the width of the ion energy distribution function through collisions between beam ions and neutral background particles. The presence of a high backpressure misrepresents the real situation in space and may adversely affect the experiments.

There are several facility effects that have significant impact on the vacuum chamber backpressure. The most significant effect is the pump absorption coefficient. In Ref. 1, different backpressures were measured with different numbers of pumps in operation and different mass flow rates from one or two thrusters. However, compared with this experimental work, there is little analytical analysis about the facility effects in the literature. An analytical study of the facility effects and the background flow will benefit numerical simulations and experiments.

The major concerns of this study are to investigate the background flow inside a vacuum chamber and to study the facility effects on this background flow. This study suggests several simple free molecular flow models by considering the fact that the background flow inside a vacuum chamber is highly rarefied. Analysis of these simple models leads to sets of analytical formulas for the background flow and the facility effects.

Section II briefly introduces background and several general assumptions. Section III presents four free molecular flow models with discussions of results. Section IV suggests an improvement of background flow treatment for numerical simulations. Section V summarizes this study.

\section{Background and General Assumptions}

\section{Background}

Vacuum chambers have different configurations based on the pump locations. One type is that both sides of pumps are exposed to background flow. Another general type is that the pumps are located at one end of the chamber and only one side of the pumps is exposed to the background flow.

The Large Vacuum Test Facility (LVTF) at the Plasmadynamics \& Electric Propulsion Laboratory in the University of Michigan belongs to the first type of vacuum chamber. It is used for studying electric plasma thrusters. In this study, the models are applied to the background flows in the LVTF. The LVTF is a stainless steel-clad vacuum chamber that has a cylindrical volume of $280 \mathrm{~m}^{3}$ with a length and diameter of $9 \mathrm{~m}$ and $6 \mathrm{~m}$. Near one end of the chamber, there are seven CVI TM-1200 nude cryopumps with a total surface area of $7.26 \mathrm{~m}^{2}$. In operation, two, four or seven pumps can be turned on, hence in different operations, the total pump area can be different. The pumps are maintained at an estimated temperature of $15 \mathrm{~K}$. When particles such as atoms or ions hit the pumps, a fraction of the particles stick to the plates and the rest rebound diffusely with a thermal speed characterized by the pump temperature of $15 \mathrm{~K}$. The pump performance is of significant importance and the pump absorption coefficient has a decisive influence on the final rarefied background flow state.

Usually there is one thruster or a cluster of thrusters mounted on a test station in front of the pumps. In the present study, Hall thrusters employing xenon propellant are considered. In operation, a low-density plasma flow is exhausted from the thruster towards the other chamber end.

Though there are ions in the plume flow field, the ion number density is far lower than the neutral number density and when these ions hit the chamber wall, they lose their charge and rebound diffusely as neutrals with a 
thermal speed characterized by the wall temperature of $300 \mathrm{~K}$. It is reasonable to assume neutrals move slowly from one chamber end towards the other end where the pumps are located.

Inside the LVTF, because the gas flow is highly rarefied, it is reasonable to separate the chamber gas pressure into two parts: a universal background pressure and a plume pressure that only exists inside the plume. The study of the first part is the primary concern in this paper.

In the experiments ${ }^{1-3}$, the backpressure of xenon is calculated using the ideal gas law $P_{b}=n k T_{w}$, where $n$ is the xenon number density measured using an ionization gauge and $T_{w}$ is the chamber temperature. The ideal location to measure the background density is on the centerline of the chamber and between the pumps and the thrusters. Locations inside the plume(s) or close to the chamber walls should be avoided because of possible plume pressure effects and wall effects.

\section{General Assumptions}

1. Pumps work fast and create a low-density environment. This assumption results in a free molecular flow at the final steady state. With a typical final xenon backpressure of $10^{-3} \mathrm{~Pa}$ in the chamber, the mean free path of xenon atoms is about $2.86 \mathrm{~m}$.

2. The chamber wall temperature is $300 \mathrm{~K}$.

3. The background flow is one-dimensional.

4. The plume flow is neglected. The reflection of plume flow from one chamber end can be considered as neutral xenon coming into the chamber through that chamber end, at the thruster mass flux rate $\dot{m}$ and wall temperature $T_{w}$. This end of the chamber is considered as a source.

5. All pumps have the same absorption coefficient $\alpha$, the same pump temperature $T_{p}$ and a total pump area $S_{p}$. Suppose the total pump area $S_{p}$ is smaller than the chamber cross section area $S_{c}$, for simplicity. When xenon atoms and ions hit one of the pumps, by a probability of $\alpha$ they stick to the pumps and by a probability of $1-\alpha$ they rebound with a thermal speed characterized by $T_{p}$. Hence the pumps can be treated as a sink. Because the flow is highly rarefied, the particles reflected from the pumps cannot hit the same pumps immediately without the necessary change of travel direction by collisions with the other chamber end or the chamber sidewalls.

With the above assumptions, the background flow in the vacuum chamber can be simplified as one free molecular flow with a source at one chamber end and a sink for the pumps on or close to the other chamber end. The source has a mass flow rate $\dot{m}$, an area $S_{c}$, a temperature $T_{w}$, and the particles hitting this source rebound diffusively with a thermal speed characterized by $T_{w}$. The sink has a temperature $T_{p}$, an area $S_{p}$ which is smaller than the chamber cross section area $S_{c}$, and the particles hitting this pump either stick to the plates by a probability of $\alpha$ or reflect diffusely with a thermal speed characterized by $T_{p}$ with a probability of $1-\alpha$.

For a rarefied gas flow in equilibrium, the velocity distribution in any coordinate direction can be described as a full Maxwellian distribution. With a temperature $T$ the velocity distribution function is:

The net mass flux in one direction across an area $S$ is:

$$
f_{1}(C) d C=(m /(2 \pi k T))^{1 / 2} \exp \left(-\frac{m}{2 k T} C^{2}\right) d C
$$

$$
\dot{m}=m n S \int_{0}^{\infty} C f_{1}(C) d C=\frac{1}{4} m n S \sqrt{(8 k T) /(\pi m)}=m n_{+} S \sqrt{(2 k T) /(\pi m)}
$$

where $\mathrm{n}_{+}=\mathrm{n} / 2$ is the number density of particles moving in one direction.

Another important relation for this study is the number density after a group of particles reflect from a plate with a different temperature. Directly from Eq. (2), to maintain the flux, the following relation must hold:

$$
n_{1} \sqrt{T_{1}}=n_{2} \sqrt{T_{2}}
$$

where the subscripts 1,2 represent incoming and reflected group of particles.

Equations (1-3) can be found in general kinetic theory books. ${ }^{4-6}$ 


\section{Free Molecular Flow Models}

\section{Model A: From Mass Conservation Law}

This model is based on the mass conservation law and is independent of the pump locations.

Assuming a constant density distribution, the mass conservation law for the gas inside the vacuum chamber is:

$$
\frac{d \rho}{d t}=\frac{d}{V d t}\left(\int \rho d v\right)=\frac{1}{V}\left(\dot{m}-\frac{1}{4} \alpha S_{p} \rho \sqrt{\frac{8 k T_{w}}{\pi m}}\right)
$$

The solution for this equation consists of one unsteady term and one steady term:

$$
\rho(t)=\rho_{0} e^{-\frac{\alpha S_{p}}{V} \sqrt{\frac{k T_{w}}{2 \pi m}} t}+\frac{\dot{m}}{\alpha S_{p}} \sqrt{\frac{2 \pi m}{k T_{w}}}
$$

The mean velocity is

$$
U(t)=\frac{\dot{m}}{S_{c} \rho(t)}=\frac{\dot{m}}{S_{c}}\left(\rho_{0} e^{-\frac{\alpha S_{p}}{V} \sqrt{\frac{k T_{w}}{2 \pi n}}}+\frac{\dot{m}}{\alpha S_{p}} \sqrt{\frac{2 \pi m}{k T_{w}}}\right)^{-1}
$$

The pressure corresponding to the experimental measurements is:

$$
P_{b}(t)=\rho(t) R T_{w}=\rho_{0} R T_{w} e^{-\frac{\alpha S_{p}}{V} \sqrt{\frac{k T_{w}}{2 \pi m} t}}+\frac{\dot{m} \sqrt{2 \pi R T_{w}}}{\alpha S_{p}}
$$

At steady state, the normalized pressure and the Mach number are:

$$
\begin{aligned}
& \frac{P_{b} S_{p}}{\dot{m} \sqrt{\gamma R T_{w}}}=\frac{1}{\alpha} \sqrt{\frac{2 \pi}{\gamma}} \\
& M=\frac{U}{\sqrt{\gamma R T_{w}}}=\frac{\alpha s}{\sqrt{2 \gamma \pi}}
\end{aligned}
$$

If the back pressure is known, then the pump absorption coefficient can be calculated using:

$$
\alpha=\frac{\dot{m} \sqrt{2 \pi R T_{w}}}{P_{b} S_{p}}
$$

This crude model, especially Eq. (7), relates several properties from the chamber, the pumps, the thruster and the propellant, but the pump temperature is not included. There are three conclusions from this model:

1. It is evident from Eq. (7) that if the pumps work fast, the pressure will decrease and reach a final steady state. However, it also indicates that the unsteady term will take several seconds to decay significantly. For example, with the following LVTF parameters: $V=280 \mathrm{~m}^{3}, T_{w}=300 \mathrm{~K}, S_{p}=7.26 \mathrm{~m}^{2}$, and an assumption of $\alpha=0.40$, the decaying term is:

$$
\rho_{b}(t)=\rho_{0} e^{-0.57 t}=\rho_{0} e^{-t / 1.7543}
$$

The significant term in this expression is the semi-decaying period $\tau_{d}=1.7543$ seconds. In experiments, usually the pumps operate for several hours, and steady background flows are well established. However, in particle simulations of the rarefied plasma plume flow field inside a vacuum chamber, usually it is modeled as an unsteady process with a time step around $1 \times 10^{-7}$ second. This requires at least 17.5 million time steps to reach a steady flow state. This presents a challenge to the numerical particle simulation and usually a full 3D simulation of the whole chamber flow is too expansive.

2. The background gas flows towards the pump, and the highest Mach number is $1 / \sqrt{2 \pi \gamma}$ with the parameters $\alpha=s=1$. This value is much lower than the value of a general free molecular flow: $\sqrt{8 k T /(\pi m)} / \sqrt{\gamma R T}=\sqrt{8 / \gamma \pi}$. Due to the chamber wall constrains, the highest Mach number is 4 times smaller than the normal value.

3. No matter how fast the pumps work, there is a certain amount of finite backpressure in the vacuum chamber. This backpressure is represented by the second term of Eq. (7). The same equation also indicates that for a specific chamber with fixed parameters, at the final steady state, the background pressure is proportional to the mass flux rate from the thrusters. Although this is a crude approximation, experimental measurements ${ }^{1}$ approximately support 


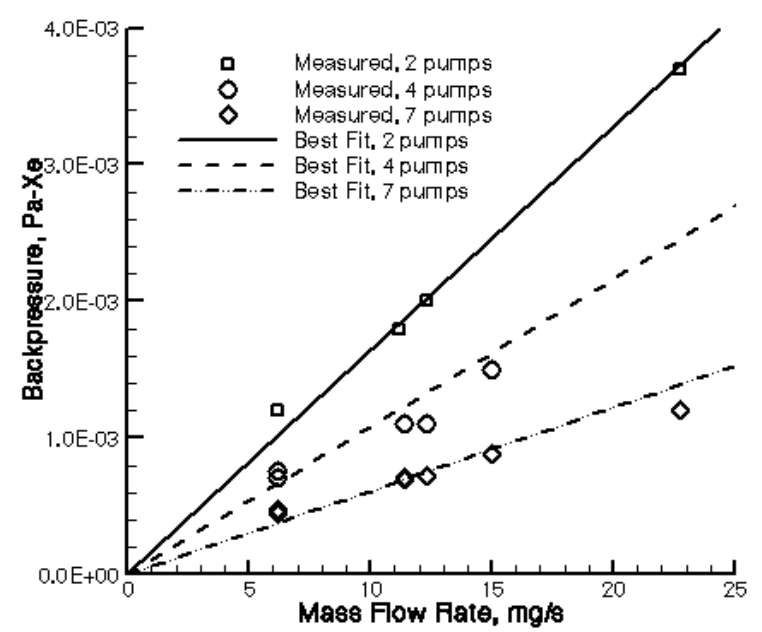

Figure 1. Flow Rate and Backpressure Measurements.
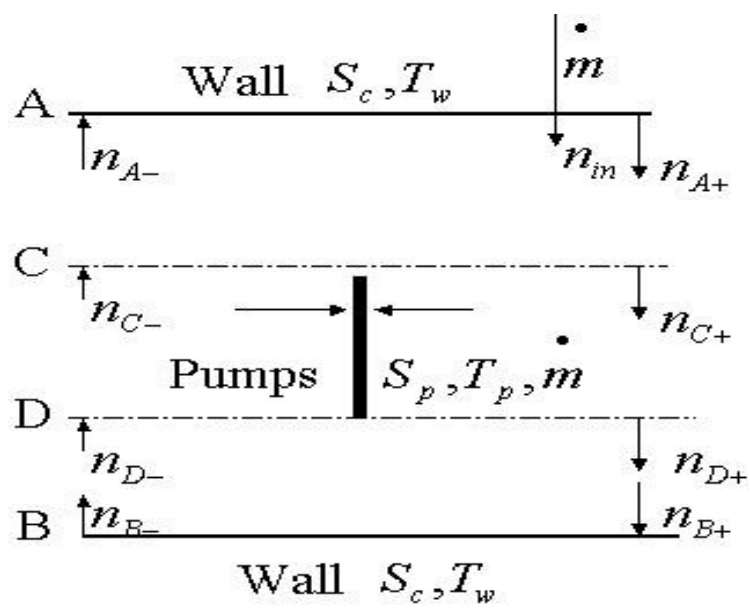

Figure 2. Illustration of Model B.

this conclusion. Different backpressures and flow rates in Ref. 1 are tabulated in Table 1 and illustrated as Fig. 1. The plot clearly displays the linear relation between backpressure and mass flow rate.

The background pressure can be calculated from Eq. (8) with a known absorption coefficient and a given mass flow rate. In general, for this model, the steady state background pressure decreases as the absorption coefficient increases. At small values of $\alpha$, a $1 \%$ difference in the coefficient may result in a significant backpressure difference, while for large values the normalized pressure is not very sensitive to this parameter. For a numerical simulation of flows inside vacuum chambers, a correct absorption coefficient is critical.

\section{Model B: Kinetic, Two-Sided Pumps}

Figure 2 illustrates the configuration of this model. Pumps are not on a chamber end and both sides of the pumps are exposed to the background flow. There is a pre-pump region AC and a post-pump region DB. The densities in these two regions are not equal. This is exactly the configuration for LVTF.

To simplify the analysis, neglect sidewall effects and suppose the reflected particles from the pumps maintain their original flow directions.

At section $\mathrm{D}$, consider the mass flux relation for the group of particles traveling from A to B:

$$
\begin{gathered}
F_{D+}=m n_{C+} S_{c} \sqrt{\frac{2 k T_{w}}{\pi m}}-m n_{C+} S_{p} \sqrt{\frac{2 k T_{w}}{\pi m}}+(1-\alpha) \sqrt{\frac{T_{w}}{T_{p}}} n_{C+} m S_{p} \sqrt{\frac{2 k T_{p}}{\pi m}} \\
n_{B+}=n_{D+}=(1-s) n_{C+}+(1-\alpha) s n_{C+} \sqrt{T_{w} / T_{p}}
\end{gathered}
$$

The second term on the right hand side represents the slow particles reflected from the pumps. These slow particles resume high speeds after they reflect from chamber end B:

$$
n_{B-}=n_{D-}=(1-s) n_{C+}+(1-\alpha) s n_{C+}=(1-\alpha s) n_{C+}
$$

The pumps further absorb a fraction of these particles:

$$
\begin{aligned}
F_{c-} & =m n_{B-} S_{c} \sqrt{\frac{2 k T_{w}}{\pi m}}-m n_{B-} S_{p} \sqrt{\frac{2 k T_{w}}{\pi m}}+(1-\alpha) \sqrt{\frac{T_{w}}{T_{p}}} n_{B-} m S_{p} \sqrt{\frac{2 k T_{p}}{\pi m}} \\
n_{A-} & =n_{C-}=(1-s) n_{B-}+(1-\alpha) s n_{B-} \sqrt{T_{w} / T_{p}}
\end{aligned}
$$

The slow particles of the second term on the right hand side resume high speeds after reflection from wall A:

$$
n_{A+}=n_{C+}=n_{i n}+(1-\alpha s) n_{B-}
$$

From Eqs. $(17,14)$, the following relation can be obtained:

$$
n_{A+}=n_{C+}=\frac{1}{\alpha s(2-\alpha s)} n_{\text {in }}
$$

The above relation can be obtained consistently with the aid of the flux in and out of the chamber as well:

$$
\dot{m}=m n_{i n} S_{c} \sqrt{\frac{2 k T_{w}}{\pi m}}=\alpha m n_{C+} S_{p} \sqrt{\frac{2 k T_{w}}{\pi m}}+\alpha m n_{B-} S_{p} \sqrt{\frac{2 k T_{w}}{\pi m}}
$$


With an intermediate result obtained from Eqs. $(16,14,18)$ :

$$
n_{A-}=n_{C-}=\frac{\left(1-s+(1-\alpha) s \sqrt{T_{w} / T_{p}}\right)(1-\alpha s)}{\alpha s(2-\alpha s)} n_{\text {in }}
$$

The final solutions for this free molecular flow model are:

$$
\begin{aligned}
& n_{A}=n_{A-}+n_{A+}=\frac{1+\left(1-s+(1-\alpha) s \sqrt{T_{w} / T_{p}}\right)(1-\alpha s)}{\alpha s(2-\alpha s)} n_{i n} \\
& n_{B}=n_{B-}+n_{B+}=\frac{2-\alpha s-s+(1-\alpha) s \sqrt{T_{w} / T_{p}}}{\alpha s(2-\alpha s)} n_{i n} \\
& \frac{2 n_{i n}}{\cos (2-\alpha s)}\left(\frac{m}{2 \pi k T}\right)^{1 / 2} \exp \left(\frac{m}{2 k T} C_{w}\right), C>0
\end{aligned}
$$

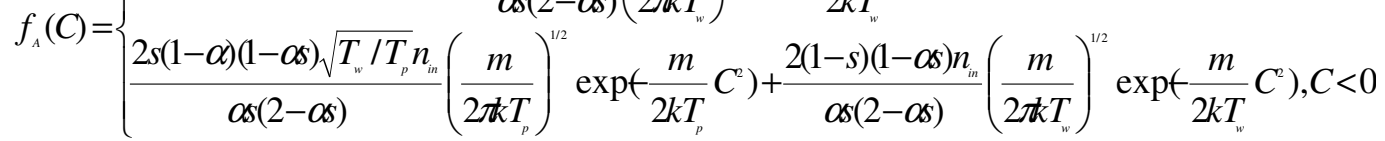

$$
\begin{aligned}
& f_{B}(C)=\left\{\begin{array}{c}
\frac{2 s(1-\alpha) \sqrt{T_{w} / T_{p}} n_{i n}}{\alpha s(2-\alpha s)}\left(\frac{m}{2 \pi k T_{p}}\right)^{1 / 2} \exp \left(-\frac{m}{2 k T_{p}} C^{2}\right)+\frac{2(1-s) n_{i n}}{\alpha s(2-\alpha s)}\left(\frac{m}{2 \pi k T_{w}}\right)^{1 / 2} \exp \left(-\frac{m}{2 k T_{w}} C^{2}\right), C>0 \\
\frac{2(1-\alpha s) n_{i n}}{\alpha s(2-\alpha s)}\left(\frac{m}{2 \pi k T_{w}}\right)^{1 / 2} \exp \left(-\frac{m}{2 k T_{w}} C^{2}\right), C<0
\end{array}\right.
\end{aligned}
$$

The normalized pressure corresponding to the experimental measurements ${ }^{1-2}$ and the mean velocities for these two regions are:

$$
\begin{gathered}
\frac{P_{b}(A) S_{p}}{\dot{m} \sqrt{\gamma R T_{w}}}=\frac{1}{\alpha(2-\alpha s)} \sqrt{\frac{\pi}{2 \gamma}}\left(1+\left(1-s+(1-\alpha) s \sqrt{\frac{T_{w}}{T_{p}}}\right)(1-\alpha s)\right) \\
\frac{P_{b}(B) S_{p}}{\dot{m} \sqrt{\gamma R T_{w}}}=\frac{1}{\alpha(2-\alpha s)} \sqrt{\frac{\pi}{2 \gamma}}\left(2-\alpha s-s+(1-\alpha) s \sqrt{\frac{T_{w}}{T_{p}}}\right) \\
\frac{U_{A}}{\sqrt{\gamma R T_{w}}}=\frac{\alpha s(2-\alpha s)}{\sqrt{\gamma \pi / 2}\left(1+\left(1-s+(1-\alpha) s \sqrt{T_{w} / T_{p}}\right)(1-\alpha s)\right)}>0 \\
\frac{U_{B}}{\sqrt{\gamma R T_{w}}}=0
\end{gathered}
$$

Several observations can be obtained based on these expressions:

1. The velocity distribution in general is not a zero-centered Maxwellian distribution. Figure 3 shows typical unbalanced profiles for several coefficient combinations.

2. The number density in the chamber varies spatially and may be affected by various effects from the chamber, the pumps, the thruster and the propellant. Figures 4 and 5 show the normalized pressure contours as a function of $s$ and $\alpha$. The temperature ratio is set to $\widetilde{T}_{p w}=15 / 300$. When $\alpha$ is very small, the model is not valid because the flow is no longer free molecular. $P_{A}$ and $P_{B}$ decrease when $\alpha$ increases. With a low absorption coefficient, an increased pump area will not always result in a lower backpressure for both ends of the chamber because more slow particles will reflect from the pump.

3. Figure 6 shows the Mach number for the pre-pump region. The velocity in the pre-pump region is always greater than zero meaning gas flows towards the pump, but the velocity in the post-pump region is always zero because of a net zero flux. The combination of $\alpha=1$ and $s=1$ will result in $n_{B}=0, M_{B}=0$, and $n_{A}=n_{i n}$, $M_{A}=\sqrt{2 /(\gamma \pi)}$. All particles hitting the pumps are absorbed and a vacuum exists in the post-pump region. Because no particles are reflected from the post-pump region, the number density at chamber end A is equal to the inlet number density.

The above discussion neglects the sidewall effects. If the sidewall is long enough, the slow particles reflected from the cold pumps will hit the sidewall first before they reach a chamber end. In a cylinder $9 \mathrm{~m}$ in length and $6 \mathrm{~m}$ in diameter, calculation shows that less than $5 \%$ of the particles from one chamber end reach the other end without any collisions on the sidewall. So, simply replacing $\sqrt{T_{p} / T_{w}}$ with one in the above formulas may approximate a long 
chamber situation. It should be pointed out that this is the most convenient and plausible treatment but not an accurate treatment. The new formulas for normalized pressure are:

$$
\begin{gathered}
\frac{P_{b}(A) S_{p}}{\dot{m} \sqrt{\gamma R T_{w}}}=\frac{2-2 \alpha s+\alpha^{2} s^{2}}{\alpha(2-\alpha s)} \sqrt{\frac{\pi}{2 \gamma}} \\
\frac{P_{b}(B) S_{p}}{\dot{m} \sqrt{\gamma R T_{w}}}=\frac{(2-2 \alpha s)}{\alpha(2-\alpha s)} \sqrt{\frac{\pi}{2 \gamma}}<\frac{P_{b}(A) S_{p}}{\dot{m} \sqrt{\gamma R T_{w}}}
\end{gathered}
$$

Because of different working conditions and measurement fluctuations, the calculated absorption coefficient for a vacuum pump may vary within a small range. Table 1 summarizes the measurements of backpressures and flow rates $^{1}$, and the absorption coefficients computed by different formulas. There are several values exceeding 1.0 from Eq. (10), which is not surprising because model A is the crudest model. For the LVTF, the pre-pump region is larger than the post-pump region and the thrusters are located in the pre-pump region. Hence the results from the pre-pump region are of primary interest. Surprisingly, the calculations for both the pre-pump region and the post-pump region yield similar results and it can be concluded that the average pump absorption coefficient for the LVTF is close to a value of 0.46 by Eqs (22a,b) or a value of 0.40 by Eqs. (23).

Figures 7-9 show three measured pressure profiles ${ }^{1}$ inside the LVTF and three series of particle simulation results with different pump absorption coefficients ${ }^{5}$. They correspond to Cases 5, 6 and 11 in Table 1. From the interpolation of computed results in Fig.7, an absorption coefficient of 0.30 0.40 may result in a match between the

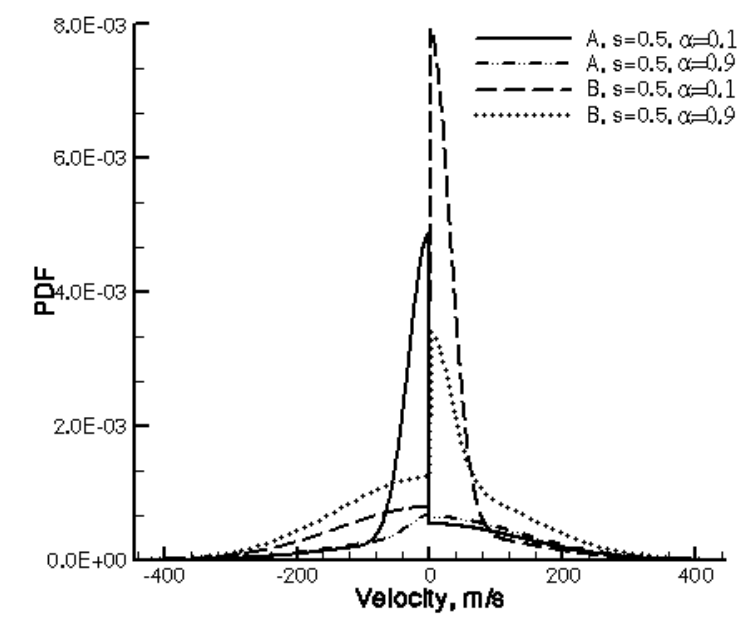

Figure 3. Velocity Distribution Examples, Eq. (21c,d)

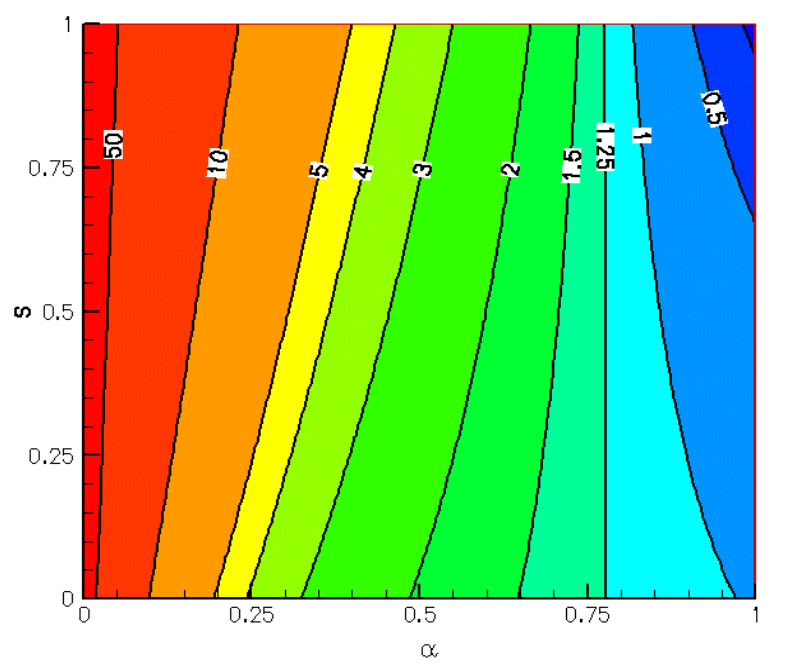

Figure 5. Normalized $P_{B}$ from Eq. (22b).

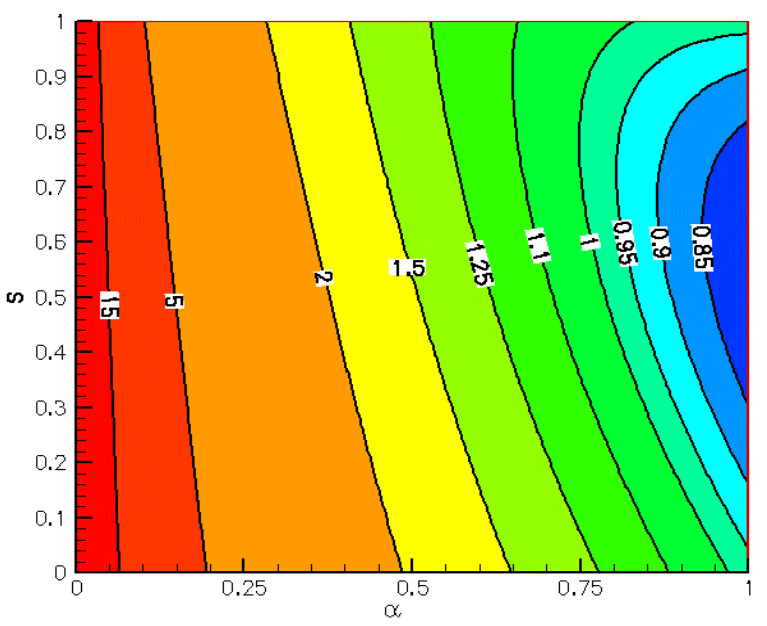

Figure 4. Normalized $P_{A}$ from Eq. (22a)

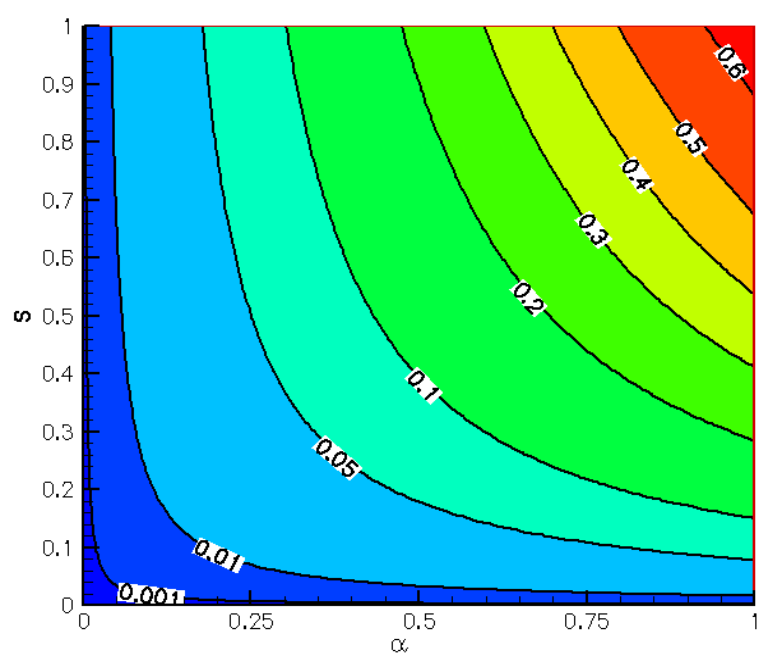

Figure 6. $M_{A}$ from Eq. (22c). 
experimental measurements and numerical simulation for Case 5, and from Table 1, the analytical formulas predict a value of 0.38 by Eqs $(22 \mathrm{a}, \mathrm{b})$ and a value of 0.33 from Eqs $(23 \mathrm{a}, \mathrm{b})$. These formulas predict very close values for Cases 6 (Fig. 8) and 11 (Fig.9) as well. Obviously the analytical predictions, especially those from Eqs (23a,b), match experimental measurement and numerical simulations very well.

It is necessary to discuss the range of validity for the above formulas. These formulas are based on a free molecular flow assumption, hence for a specific mass flux and a specific propellant, it is not difficult to evaluate whether the assumption is correct. Because the pre-pump region is the major concern, the number density $n_{A}$ and the chamber end radius are selected as the characteristic quantities for the evaluation of Knudsen number:

$$
K n=\frac{\lambda}{\sqrt{S_{C} / \pi}}=\frac{1}{\sqrt{2 \pi} d^{2} \sqrt{S_{C}} n_{A}}=\frac{\cos (2-\alpha s) m \sqrt{R T_{w} S_{C}}}{\pi d^{2} \dot{m}\left(1+\left(1-s+(1-\alpha) s \sqrt{T_{p} / T_{w}}\right)(1-\alpha s)\right)}
$$

where the absorption coefficient in this formula should be computed with Eq. (22a) if not given as a known quantity.
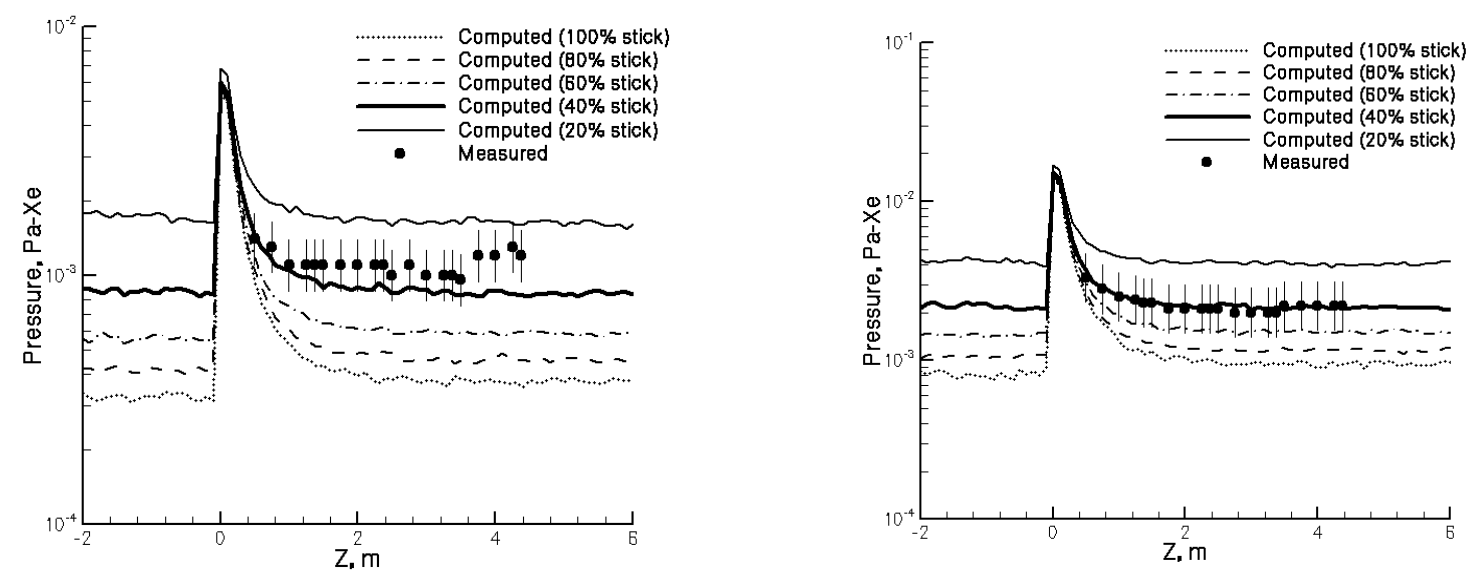

Figure 7. Comparisons of Simulated and Measured Figure 8. Comparisons of Simulated and Measured Pressure Distribution within LVTF with cold flow Pressure Distribution within LVTF with cold flow rate rate $=5.25 \mathrm{mg} / \mathrm{s}, 4$ pumps and 1 thruster in operation. $=10.46 \mathrm{mg} / \mathrm{s}, 4$ pumps and 1 thruster in operation.

From the tabulated values of Knudsen number in Table 1, it can be observed that the cases with two pumps in operation are not quite free molecular as they do not satisfy $K n>1$.

The final comment about this model is to note that Eqs. (12-23) are obtained with an assumption of onedimension flow, and the model is essentially a constant density model. Equations. (23a,b) are extrapolations of this 1D model. More accurate results should be obtained with careful evaluations of the flux relations and different solid angles as will be presented in model D. Because model B consists of two regions and the derivation is significantly more complex than model D, the details are omitted here. However, Figs.7-9 indicate that this simpler analytical 1-D model yields very good agreement with the numerical simulations.

\section{Model C: Kinetic, One-Sided Pumps}

Another general configuration for a vacuum chamber has the pumps located on one end and only one side of the pumps are exposed to the background flow, as illustrated by Fig. 10. This is a less efficient pumping configuration than the previous one. Though this is not the case of the LVTF, it is another standard free molecular flow problem deserving discussion to complete this study.

At the first step, model C still assumes a constant density distribution. In the next model, this assumption will be dropped.

At end A, a group of particles moves from A to B with a number density $n_{A+}=n_{i n}+n_{A 1+}+n_{A 2+}$.

At end $\mathrm{B}$, there are three number density relations from the flux relations:

1. Particles reflected at end B but not from the pumps:

$$
n_{B 2-}=n_{A+}\left(1-\frac{S_{p}}{S_{c}}\right)=n_{A+}(1-s)
$$

2. Mass flux relation: flux out of the chamber through the pumps equals the flux into the chamber at chamber end A: 
3. Particles reflected from the pumps:

$$
\dot{m}=\alpha m n_{A+} S_{p} \sqrt{\frac{2 k T_{w}}{\pi m}}=m n_{i n} S_{C} \sqrt{\frac{2 k T_{w}}{\pi m}}
$$

$$
n_{B 1-} \sqrt{T_{p}}=(1-\alpha) n_{A+} \frac{S_{p}}{S_{c}} \sqrt{T_{w}}=(1-\alpha) s n_{A+} \sqrt{T_{w}}
$$

The above relations yield the following expressions:

$$
\begin{gathered}
n_{A 1-}=n_{B 1-}=\frac{(1-\alpha) \sqrt{T_{w} / T_{p}} n_{i n}}{\alpha} \\
n_{A 2+}=n_{A 2-}=n_{B 2-}=\frac{(1-s) n_{i n}}{\alpha s} \\
n_{A 1+}=n_{B 1-} \sqrt{T_{p} / T_{w}}=\frac{(1-\alpha) n_{i n}}{\alpha} \\
n_{A+}=\frac{n_{i n}}{\alpha S}
\end{gathered}
$$

With the above relations, the full solutions for this model are:

$$
\begin{array}{r}
n_{A}=n_{B}=n_{A+}+n_{A 1-}+n_{A 2-}=\left(\frac{2-s+s(1-\alpha) \sqrt{T_{w} / T_{p}}}{\alpha s}\right) n_{i n}=\frac{\dot{m}}{S_{c}} \sqrt{\frac{\pi}{2 k m T_{w}}}\left(\frac{2-s+s(1-\alpha) \sqrt{T_{w} / T_{p}}}{\alpha s}\right) \\
f(C)=\left\{\begin{array}{l}
\frac{2 n_{i n}}{\alpha s}\left(\frac{m}{2 \pi k T_{w}}\right)^{1 / 2} \exp \left(-\frac{m}{2 k T_{w}} C^{2}\right), C>0 \\
\frac{2(1-\alpha) s \sqrt{T_{w} / T_{p}} n_{i n}}{\alpha s}\left(\frac{m}{2 \pi k T_{p}}\right)^{1 / 2} \exp \left(-\frac{m}{2 k T_{p}} C^{2}\right)+\frac{2(1-s) n_{i n}}{\alpha s}\left(\frac{m}{2 \pi k T_{w}}\right)^{1 / 2} \exp \left(-\frac{m}{2 k T_{w}} C^{2}\right), C<0
\end{array}\right.
\end{array}
$$

The normalized mean velocity, pressure corresponding to experimental measurements, pump absorption coefficient, and Knudsen number are:

$$
\begin{gathered}
\frac{U}{\sqrt{\gamma R T_{w}}}=\frac{\sqrt{2} s \alpha}{\sqrt{\gamma \pi}\left(2-s+s(1-\alpha) \sqrt{T_{w} / T_{p}}\right)} \\
\frac{P_{b} S_{p}}{\dot{m} \sqrt{\gamma R T_{w}}}=\sqrt{\frac{\pi}{2 \gamma}\left(\frac{2-s+s(1-\alpha) \sqrt{T_{w} / T_{p}}}{\alpha}\right)}
\end{gathered}
$$

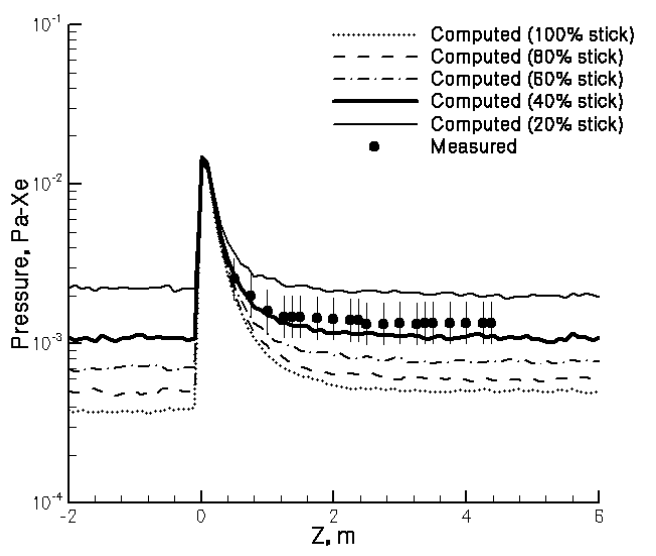

Figure 9. Comparisons of Simulated and Measured Pressure Distribution within LVTF with cold flow rate $=10.46 \mathrm{mg} / \mathrm{s}$, 7 pumps and 1 thruster in operation.
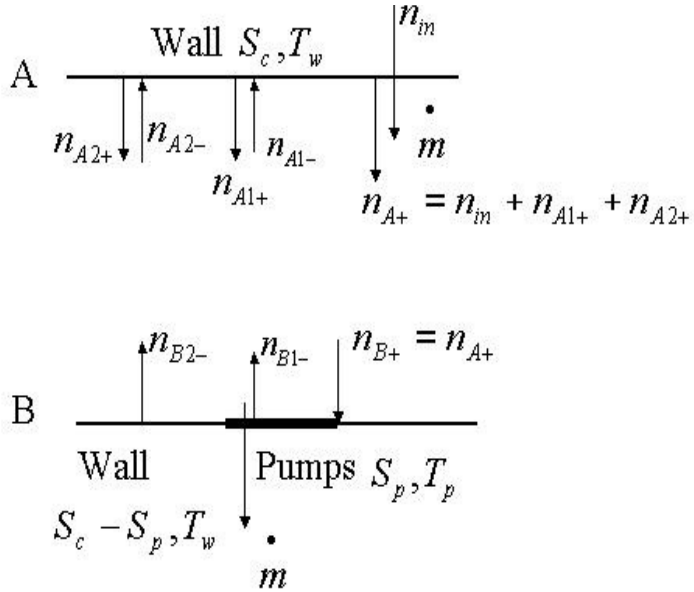

Figure 10. Illustration of Model C. 


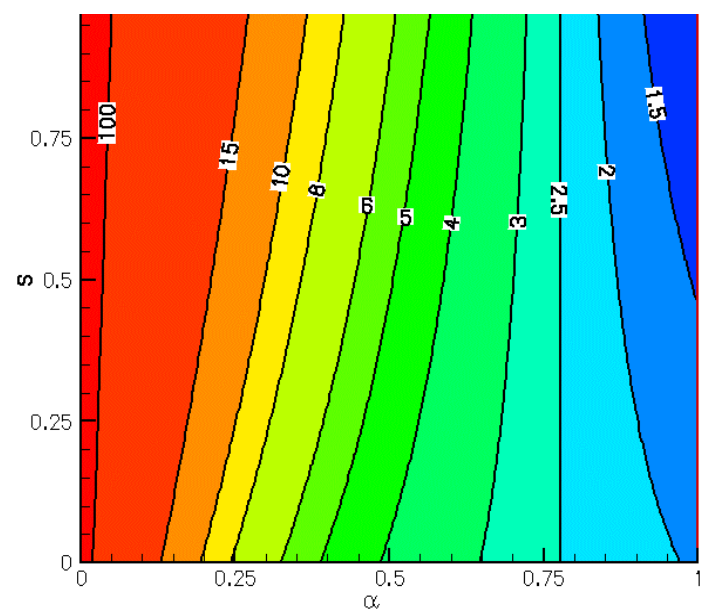

Figure 11. Normalized Pressure for Model C,

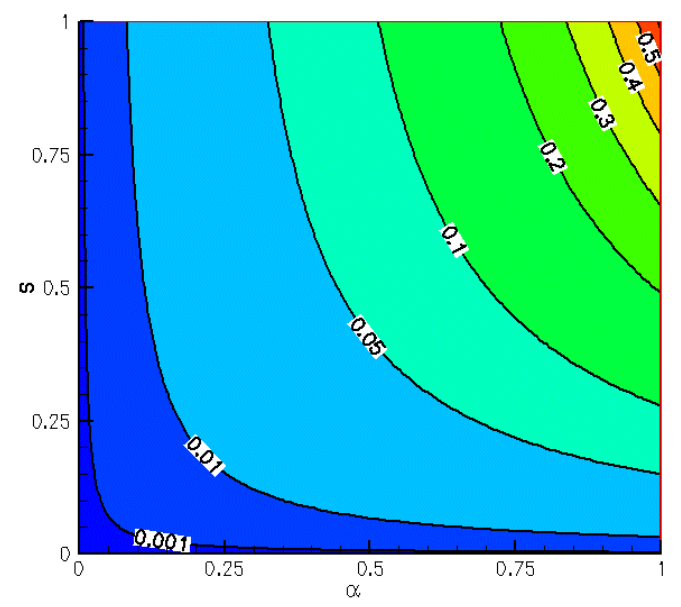

Figure 12. Mach Number for Model C, Eq. (33b).

Eq. (33a).

$$
\begin{gathered}
\alpha=\frac{2-s+s \sqrt{T_{w} / T_{p}}}{s \sqrt{\frac{T_{w}}{T_{p}}}+\frac{P_{b} S_{p}}{\dot{m} \sqrt{\pi R T_{w} / 2}}} \\
K n=\frac{\lambda}{\sqrt{S_{C} / \pi}}=\frac{1}{\sqrt{2 \pi} d^{2} \sqrt{S_{C} n_{A}}}=\frac{\alpha s m \sqrt{R T_{w} S_{C}}}{\left.\pi d^{2} \dot{m}\left(2-s+(1-\alpha) s \sqrt{T_{w} / T_{p}}\right)\right)}
\end{gathered}
$$

Both model B and model $\mathrm{C}$ are exact solutions to classical free molecular flow problems. Comparing these two sets of results, it is evident that the two models perform very differently. Generally, a vacuum system consistent with model $\mathrm{C}$ works less efficiently since only the particles moving towards end $\mathrm{B}$ have a probability to be absorbed. If the formulas from model $\mathrm{C}$ are used to calculate the pump absorption coefficient for the LVTF, the computed results will be two times higher than the results from model B for the same back pressure.

Figure 11 shows contours of the normalized pressure inside the chamber. The temperature ratio is set to $T_{P} / T_{w}$ $=15 / 300$. This figure indicates that when the absorption coefficient is smaller than a threshold value, an increase of pump area results in a net pressure increase because more slow particles are reflected back from the pump to the other end.

Figure 12 shows contours of Mach number in the chamber with the temperature ratio set to $T_{P} / T_{w}=15 / 300$. The mean flow direction is always towards the pump and the mean velocity increase as $\alpha$ or $s$ increases. The highest Mach number is $\sqrt{2 /(\pi \gamma)}$, the same as in model B. Due to the constraints from the chamber, the highest Mach number is 2 times smaller than the average Mach number of a free molecular flow.

Model $\mathrm{C}$ is a constant density model and a special case of the next general model.

\section{Kinetic, One-Sided Pumps}

Different from the previous treatment of considering sidewall effects by dropping the temperature ratio, the following non-constant density model considers sidewall effects generally. Figure 13 illustrates this model.

First from the relation of the mass flow into the chamber at end A and that out of the chamber through the

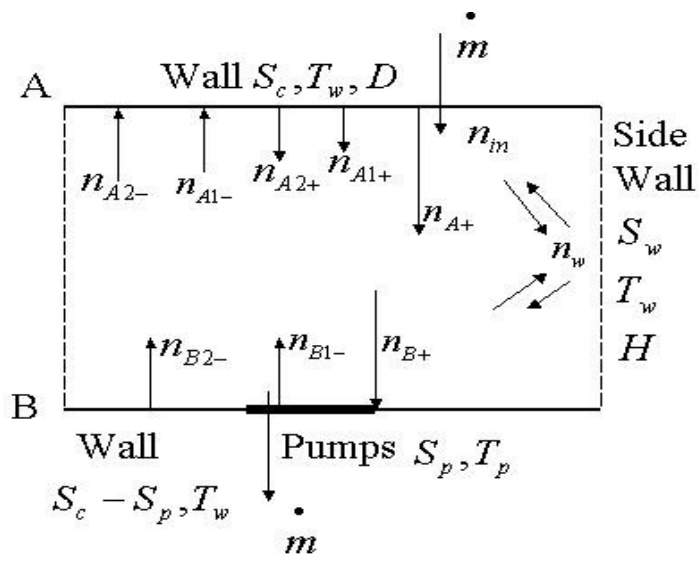

Figure 13. Illustration of Model D. pump:

the following relation is obtained:

$$
\dot{m}=m n_{i n} S_{C} \sqrt{\frac{2 k T_{w}}{\pi m}}=\alpha m n_{B+} S_{p} \sqrt{\frac{2 k T_{w}}{\pi m}}
$$




$$
n_{B+}=\frac{n_{i n}}{\alpha S}
$$

At chamber end $\mathrm{B}$, the following relations exist for the mass flux relations towards the pumps and other regions of end B:

$$
\begin{gathered}
n_{B 1-}=(1-\alpha) s \sqrt{T_{w} / T_{p}} n_{B+}=(1 / \alpha-1) \sqrt{T_{w} / T_{p}} n_{i n} \\
n_{B 2-}=(1-s) n_{B+}=\frac{(1-s) n_{i n}}{\alpha s}
\end{gathered}
$$

At both ends, the incoming particles are composed of two groups: one group of particles travel from the other end without any collisions with the sidewalls, while the other group of particles travel from the sidewall. Denote these two transportation factors as $\beta$ and $\gamma$. Further assuming the particles close to the sidewall may drift to both ends with equal probability because of the diffuse wall reflection assumption, the following equations are obtained:

$$
\begin{gathered}
n_{B+}=\beta n_{A+}+\gamma n_{w} \\
n_{A+}=n_{i n}+\gamma n_{w}+\beta n_{B 2-}+\beta n_{B 1-} \sqrt{T_{p} / T_{w}}
\end{gathered}
$$

Eqs. (35-38) yield the following intermediate results:

$$
\begin{gathered}
n_{A+}=\frac{(1+\beta+\alpha s-\alpha \beta s) n_{i n}}{\alpha s(1+\beta)} \\
n_{A-}=\beta n_{B 1-}+\beta n_{B 2-}+m_{w}=\frac{\left(\beta s(1-\alpha)(\beta+1) \sqrt{T_{w} / T_{p}}+1+\beta-\beta s-\beta^{2} s-\alpha \beta s+\alpha \beta^{2} s\right) n_{i n}}{\alpha s(1+\beta)}
\end{gathered}
$$

The complete solutions of this model involve an extra geometry parameter $\beta$, which is determined by the chamber length and the chamber base radius:

$$
\begin{aligned}
& n_{A}=\frac{\left(\beta s(1-\alpha)(\beta+1) \sqrt{T_{w} / T_{p}}+2+2 \beta+\alpha s-\beta s-\beta^{2} s-2 \alpha \beta+\alpha \beta^{2} s\right) n_{i n}}{\operatorname{cs}(\beta+1)} \\
& n_{B}=\frac{\left((1-\alpha) s \sqrt{T_{w} / T_{p}}+(2-s)\right) n_{i n}}{\alpha s} \\
& \frac{2(1+\beta+\alpha s-\alpha \beta s) n_{i n}}{\alpha s(\beta+1)}\left(\frac{m}{2 \pi k T_{w}}\right)^{1 / 2} \exp \left(-\frac{m}{2 k T_{w}} C^{2}\right), C>0 \\
& f(A)=\left\{\frac{2 \beta s(1-\alpha)(\beta+1) \sqrt{T_{w} / T_{p}} n_{i n}}{\alpha s(\beta+1)}\left(\frac{m}{2 \pi k T_{p}}\right)^{1 / 2} \exp \left(-\frac{m}{2 k T_{p}} C^{2}\right)+\right. \\
& \frac{2\left(1+\beta-\beta s-\beta^{2} s-\alpha \beta s+\alpha \beta^{2} s\right) n_{i n}}{\alpha s(\beta+1)}\left(\frac{m}{2 \pi k T_{w}}\right)^{1 / 2} \exp \left(-\frac{m}{2 k T_{w}} C^{2}\right), C<0 \\
& f(B)=\left\{\begin{array}{c}
\frac{2 n_{i n}}{\alpha s}\left(\frac{m}{2 \pi k T_{w}}\right)^{1 / 2} \exp \left(-\frac{m}{2 k T_{w}} C^{2}\right), C>0 \\
\frac{2 s(1-\alpha) \sqrt{T_{w} / T_{p}} n_{i n}}{\alpha s}\left(\frac{m}{2 \pi k T_{p}}\right)^{1 / 2} \exp \left(-\frac{m}{2 k T_{p}} C^{2}\right)+\frac{2(1-s) n_{i n}}{\alpha s}\left(\frac{m}{2 \pi k T_{w}}\right)^{1 / 2} \exp \left(-\frac{m}{2 k T_{w}} C^{2}\right), C<0
\end{array}\right.
\end{aligned}
$$

The normalized mean velocities, the normalized pressures corresponding to measurements and the Kundsen number are:

$$
\begin{gathered}
\frac{U(A)}{\sqrt{\gamma R T_{w}}}=\frac{\sqrt{2} \alpha s(1+\beta)}{\sqrt{\gamma \pi}\left(\beta s(1-\alpha)(\beta+1) \sqrt{T_{w} / T_{p}}+2+2 \beta+\alpha s-\beta s-\beta^{2} s-2 \alpha \beta s+\alpha \beta^{2} s\right)}>0 \\
\frac{U(B)}{\sqrt{\gamma R T_{w}}}=\frac{\sqrt{2} \alpha s}{\sqrt{\gamma \pi}\left(2-s+(1-\alpha) s \sqrt{T_{w} / T_{p}}\right)}>0
\end{gathered}
$$




$$
\begin{aligned}
& \frac{P_{b}(A) S_{p}}{\dot{m} \sqrt{\gamma R T_{w}}}=\sqrt{\frac{\pi}{2 \gamma}}\left(\frac{\beta s(1-\alpha)(\beta+1) \sqrt{T_{w} / T_{p}}+2+2 \beta+\alpha s-\beta s-\beta^{2} s-2 \alpha \beta s+\alpha \beta^{2} s}{\alpha(\beta+1)}\right) \\
& \frac{P_{b}(B) S_{p}}{\dot{m} \sqrt{\gamma R T_{w}}}=\sqrt{\frac{\pi}{2 \gamma}}\left(\frac{2-s+(1-\alpha) s \sqrt{T_{w} / T_{p}}}{\alpha}\right) \\
& K n=\frac{\lambda}{\sqrt{S_{C} / \pi}}=\frac{1}{\sqrt{2 \pi} d^{2} \sqrt{S_{C}} n_{A}}=\frac{\alpha s(1+\beta) m \sqrt{R T_{w} S_{C}}}{\pi d^{2} \dot{m}\left(\beta s(1-\alpha)(\beta+1) \sqrt{T_{w} / T_{p}}+2+2 \beta+\alpha s-\beta s-\beta^{2} s-2 \alpha \beta s+\alpha \beta^{2} s\right)}
\end{aligned}
$$

There are several significant observations from the above relations:

1. The formulas for chamber end B with pumps are the same as the results from Model C.

2. Generally the formulas for A and B are different, and a non-constant density distribution occurs.

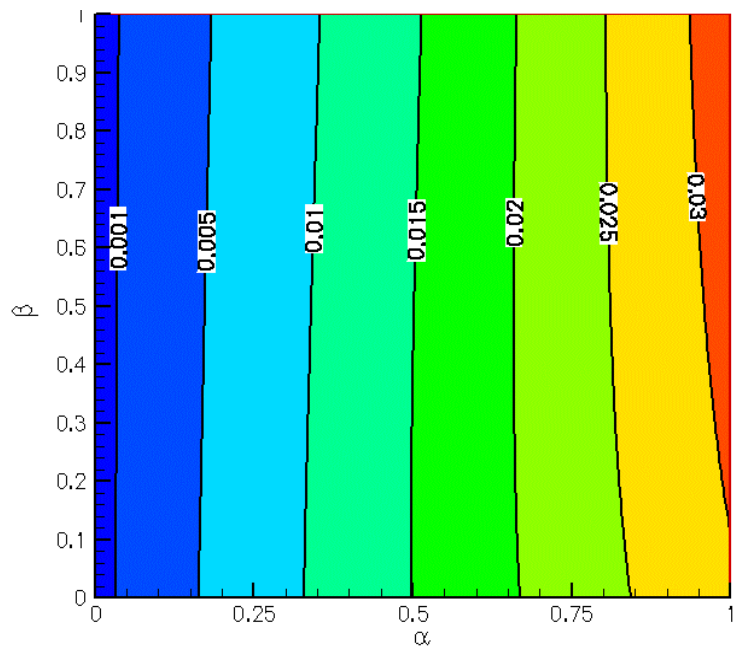

Figure 14. Mach Number M(A), Model D, Eq.(41a). $\left(\mathrm{T}_{\mathrm{w}} / \mathrm{T}_{\mathrm{p}}=300 / 15, s=0.5\right)$

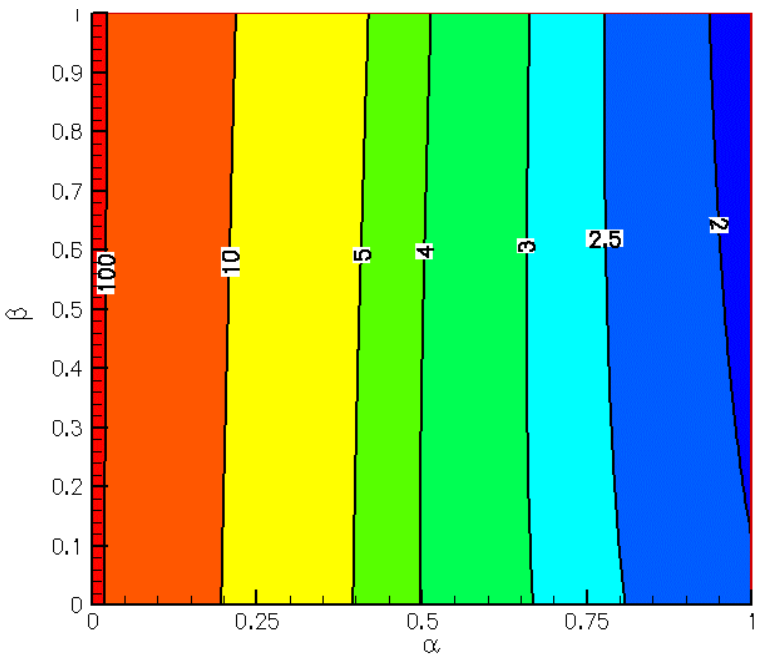

Figure 15. Normalized Pressure P(A), Model D, Eq.(41c). $\left(\mathrm{T}_{\mathrm{w}} / \mathrm{T}_{\mathrm{p}}=300 / 15, s=0.5\right)$.

3. The background gas flows towards the pump.

4. This model can be considered as a finite chamber length model for consideration of the second pump. The constant density model $\mathrm{C}$ is a special case of this model. When $\beta=1$, Eqs. (41) degenerate to the constant density distribution of model $\mathrm{C}$.

5. The highest Mach number is the same as Model C, and happens at both ends: $M=\sqrt{2 /(\gamma \pi)}$ with $\alpha=\beta=\mathrm{s}=1$.

Figures 14 and 15 show contours of Mach number and normalized pressure for end A. The ratio $s$ of pump area and the chamber cross section area is set to 0.5 and the temperature ratio is $\mathrm{T}_{\mathrm{w}} / \mathrm{T}_{\mathrm{P}}=300 / 15$. For a fixed $\alpha$, a larger $\beta$ represents a shorter chamber length. The same as in the previous models, an increase in pumps area does not always result in a lower pressure at end $\mathrm{A}$.

The last necessary information for this model is the transport coefficient $\beta$, which controls the probability that a particle traveling from one chamber end can reach the other chamber end without any collisions with the chamber sidewall. The evaluation of this parameter involves two steps: first, calculate the solid angles at one specific point on one chamber end subtended by the other chamber end. The solid angle formula is:

$$
\Omega=\int_{s} \frac{\vec{r} \bullet \vec{n} d S}{r^{3}}=\int_{0}^{2 \pi} H d \varphi \int_{0}^{R} \frac{\eta^{\prime 2} d \eta^{\prime}}{\left(r^{2}+H^{2}-2 r \eta^{\prime} \cos \varphi+\eta^{\prime 2}\right)^{3 / 2}}
$$

The final format of this integral involves the Heuman's Lambda function and the complete elliptic integral of the first kind. ${ }^{8,9}$ The second step is to average the solid angles over all points on this chamber end.

This study concerns the averaged solid angle and by a computer evaluation the average transport coefficient $\beta$ is equal to 0.044 for the LVTF.

\section{Impacts on Numerical Simulation}

The analytical results have significant effects for particle simulations of plume flows inside a vacuum chamber. 
To simulate a rarefied plume flow inside a vacuum chamber, usually the direct simulation Monte Carlo method ${ }^{4}$ is adopted and an axi-symmetric simplification substitutes an expensive full-scale 3D simulation. Traditionally the background flow effects are either omitted or approximated by a few static background particles in each cell. The velocities of these static background particles are randomly assigned from a zero-centered Maxwellian velocity distribution.

However, from the above discussions, it is demonstrated that essentially there are 3 groups of particles inside a vacuum chamber: two groups diffuse from the end with the pumps, while the other diffuses from the other chamber end, and the velocities for the background particles are highly affected by various factors. The plume flow inside a vacuum chamber is more like firing a plume flow into another uniform background flow towards the pump. The velocity distributions for the rarefied background flow is far from a full Maxwellian distribution, the mean velocity of the background flow is far from zero and can reach a Mach number $\sqrt{2 /(\gamma \pi)}$, which is over 0.6 for xenon.

To accurately account for the rarefied background flow, there are several options:

1. Present the backpressure by background static particles, but assign the background particles with velocities sampled from the analytical velocity distribution functions obtained in this study.

2. Consider the background flow with inflow boundary conditions, neglect background particles. The front and backsides of the simulation domain use different sides of the Maxwellian distributions obtained in this study.

3. Consider the background flow with a general inflow boundary condition for all sides. This approach will need to calculate a new number density, a new mean velocity and a new temperature from the velocity distributions obtained in this study, and reconstruct a new Maxwellain distribution with the above information.

\section{Conclusions}

In this study, four different free molecular flow models were presented for the rarefied background flow inside a vacuum chamber.

The first model is a crude model with a strong assumption of a constant density distribution inside the vacuum chamber, and it leads to a set of general but crude solutions.

The second model is a free molecular flow model in which both sides of the pumps are exposed to the background flow. Analyzing the flux relation led to a detailed velocity distribution for the background flow, and based on this distribution, the mean velocity and pressure were obtained. With the experimental measurement in Ref. 1, the LVTF pump absorption coefficients were calculated. On average the coefficient is 0.39 , while for different working conditions, the coefficient can fluctuate within a small range. Compared with numerical simulations of specific flows inside the LVTF, the analytical formulas predicted a very similar absorption coefficient.

The third and fourth models were two free molecular flow models for a different configuration in which the pumps are located at one chamber end and only one side was exposed to the background flow. This configuration was less efficient than the second model since the pumps only absorb particles traveling from one direction. The third model was a constant density model and it could be considered as a special case of the fourth model. The fourth model is a non-constant density model that includes all wall effects in a transport coefficient $\beta$.

The solutions of these models were four sets of analytical formulas, including comprehensive velocity distribution functions, and the pump absorption coefficient can be computed with these formulas. These formulas connect different properties from the pumps, the chamber, the propellant and the thruster. They are applicable to evaluate or predict the performance of a vacuum chamber.

These models indicated that the background flow in a chamber was different from vacuum, and it cannot be simply treated as a static gas field. The background gas always flows to the pump with a reduced maximum Mach number. The extreme Mach number reached is 0.6 for xenon and the velocity distribution function can deviate far from a Maxwellian distribution.

The formulas for the background gas obtained from this study can be used to establish a correct background flow field for numerical simulations.

It is worthy to mention that these models and formulas are applicable to other vacuum chambers with different applications such as materials processing.

$\underline{-13-}$

American Institute of Aeronautics and Astronautics Paper 2005-4660 


\section{Acknowledgements}

The work is partially supported by the Air Force Office of Scientific Research through Grant F49620-03-1-0123. The authors gratefully acknowledge the contributions to this work by Dr. Mitchell Walker for sharing his experimental data.

\section{References}

${ }^{1}$ Walker, M.L., "Effects of Facility Backpressure on the Performance and Plume of a Hall Thruster," Ph.D. Dissertation, Aerospace Engineering Dept., the Univ. of Michigan, Ann Arbor, MI, 2005.

${ }^{2}$ Beal, B.E., "Clustering of Hall Effect Thrusters for High-Power Electric Propulsion," Ph.D. Dissertation, Aerospace Engineering Dept., the Univ. of Michigan, Ann Arbor, MI, 2004.

${ }^{3}$ Hargus, W.A. and Reed, G., "The Air Force Clustered Hall Thruster Program", AIAA Paper 2002-3678, July 2002, Indianapolis, IN.

${ }^{4}$ Bird, G.A., Molecular Gas Dynamics and the Direct Simulation of Gas Flows, Clarendon Press, New York, 1991.

${ }^{5}$ Gombosi, T., Gaskinetic Theory, Cambridge University Press, New York, 1994.

${ }^{6}$ Chen, X., Gas Dynamics and Applications in Heat Transfer and Flow, Tsinghua University Press, Beijing, 1996.

${ }^{7}$ Walker,M.L., Gallimore, A.D., Boyd, I.D. and Cai, C., "Vacuum Chamber Pressure Maps of a Hall Thruster Cold-Flow Expansion", Journal of Propulsion and Power, Vol. 20, No. 6, pp1127-1131, November-December, 2004.

${ }^{8}$ Narasimha, R., “Orifice Flow of High Knudsen Number”, Journal of Fluid Mechanics, 1961, October, pp.371-384.

${ }^{9}$ Narasimha, R., "Some Flow Problems in Rarefied Gas Dynamics", Ph.D. Dissertation, California Institute of Technology, Pasadena, CA, 1961.

Table 1. Measured Backpressure and Absorption Coefficients for the LVTF, PEPL (H: hot flow, C: cold flow. For all cases the cathode flux $=0.92 \mathrm{mg} / \mathrm{s}$ )

\begin{tabular}{|c|c|c|c|c|c|c|c|c|c|c|c|}
\hline No. & Pumps & Thrusters & $\begin{array}{c}\text { Anode Flux } \\
(\mathrm{mg} / \mathrm{s})\end{array}$ & $\begin{array}{c}\text { Total Flux } \\
(\mathrm{mg} / \mathrm{s})\end{array}$ & $\begin{array}{c}\text { Pressure } \\
(\text { Pa-Xe) }\end{array}$ & $\begin{array}{c}\alpha \\
(\text { Eq.10) }\end{array}$ & $\begin{array}{c}\alpha \\
(\text { Eq.22a) }\end{array}$ & $\begin{array}{c}\alpha \\
(\text { Eq.22b) }\end{array}$ & $\begin{array}{c}\alpha \\
(\text { Eq.23a) }\end{array}$ & $\begin{array}{c}\alpha \\
(\text { Eq.23b) }\end{array}$ & $\begin{array}{c}\text { Kn } \\
(\text { Eq.24) }\end{array}$ \\
\hline 1 & 2 & 1 & $5.25(\mathrm{H})$ & 6.17 & $1.2 \mathrm{e}-3$ & 0.8563 & 0.4531 & 0.4500 & 0.4218 & 0.4208 & 0.7849 \\
\hline 2 & 2 & 1 & $10.24(\mathrm{H})$ & 11.16 & $1.8 \mathrm{e}-3$ & 1.0300 & 0.5361 & 0.5367 & 0.5062 & 0.5068 & 0.5246 \\
\hline 3 & 2 & 2 & $5.25(\mathrm{H})$ & 12.34 & $2.0 \mathrm{e}-3$ & 1.0276 & 0.5331 & 0.5350 & 0.5042 & 0.5041 & 0.4728 \\
\hline 4 & 2 & 2 & $10.46(\mathrm{H})$ & 22.76 & $3.7 \mathrm{e}-3$ & 1.0245 & 0.5321 & 0.5322 & 0.5021 & 0.5028 & 0.2549 \\
\hline 5 & 4 & 1 & $5.25(\mathrm{C})$ & 6.17 & $7.6 \mathrm{e}-4$ & 0.6760 & 0.3817 & 0.3820 & 0.3305 & 0.3290 & 1.2469 \\
\hline 6 & 4 & 1 & $10.46(\mathrm{C})$ & 11.38 & $1.1 \mathrm{e}-3$ & 0.8615 & 0.4730 & 0.4748 & 0.4180 & 0.4172 & 0.8630 \\
\hline 7 & 4 & 1 & $14.09(\mathrm{C})$ & 15.01 & $1.5 \mathrm{e}-3$ & 0.8333 & 0.4580 & 0.4610 & 0.4041 & 0.4041 & 0.6321 \\
\hline 8 & 4 & 1 & $5.25(\mathrm{H})$ & 6.17 & $7.1 \mathrm{e}-4$ & 0.7236 & 0.4050 & 0.4061 & 0.3527 & 0.3520 & 1.3342 \\
\hline 9 & 4 & 2 & $5.25(\mathrm{H})$ & 12.34 & $1.1 \mathrm{e}-3$ & 0.9342 & 0.5061 & 0.5009 & 0.4522 & 0.4512 & 0.8461 \\
\hline 10 & 7 & 1 & $5.25(\mathrm{C})$ & 6.17 & $4.7 \mathrm{e}-4$ & 0.6247 & 0.3800 & 0.3862 & 0.3011 & 0.3009 & 2.0472 \\
\hline 11 & 7 & 1 & $10.46(\mathrm{C})$ & 11.38 & $6.9 \mathrm{e}-4$ & 0.7848 & 0.4590 & 0.4670 & 0.3748 & 0.3733 & 1.3951 \\
\hline 12 & 7 & 1 & $14.09(\mathrm{C})$ & 15.01 & $8.8 \mathrm{e}-4$ & 0.8116 & 0.4730 & 0.4792 & 0.3867 & 0.3847 & 1.0920 \\
\hline 13 & 7 & 1 & $5.25(\mathrm{H})$ & 6.17 & $4.5 \mathrm{e}-4$ & 0.6524 & 0.3944 & 0.4012 & 0.3122 & 0.3125 & 2.1419 \\
\hline 14 & 7 & 1 & $5.25(\mathrm{H})$ & 6.17 & $4.6 \mathrm{e}-4$ & 0.6382 & 0.3870 & 0.3911 & 0.3070 & 0.3062 & 2.0782 \\
\hline 15 & 7 & 1 & $10.46(\mathrm{H})$ & 11.38 & $7.1 \mathrm{e}-4$ & 0.7627 & 0.4728 & 0.4780 & 0.3637 & 0.3620 & 1.0881 \\
\hline 16 & 7 & 2 & $5.25(\mathrm{H})$ & 12.34 & $7.2 \mathrm{e}-4$ & 0.8155 & 0.4740 & 0.4810 & 0.3892 & 0.3865 & 1.3344 \\
\hline 17 & 7 & 2 & $10.46(\mathrm{H})$ & 22.76 & $1.2 \mathrm{e}-3$ & 0.9025 & 0.5150 & 0.5221 & 0.4272 & 0.4252 & 0.8012 \\
\hline Ave & & & & & & 0.8210 & $\mathbf{0 . 4 6 0 8}$ & $\mathbf{0 . 4 6 3 7}$ & $\mathbf{0 . 3 9 6 5}$ & $\mathbf{0 . 3 9 6 4}$ & \\
\hline
\end{tabular}

